American Journal of Applied Sciences 3 (4): 1803-1809, 2006

ISSN 1546-9239

(C) 2006 Science Publications

\title{
Optimal Parameter Tuning in a Predictive Nonlinear Control Method for a Mobile Robot
}

\author{
${ }^{1}$ D. Hazry and ${ }^{2} \mathrm{M}$. Sugisaka \\ ${ }^{1}$ Artificial Life and Robotics Laboratory, Department of Electrical and Electronic Engineering \\ Oita University, 700 Oaza Dannoharu, Oita 870-1192, Japan \\ ${ }^{2}$ Department of Electrical and Electronic Engineering, Oita University \\ 700 Oaza Dannoharu, Oita 870-1192, Japan \\ ${ }^{2}$ Advance Research Institute for Science and Engineering, Waseda University, 2-2 Hibikino \\ Wakamatsu, Kitakyushu, Fukuoka 808-0135, Japan
}

\begin{abstract}
This study contributes to a new optimal parameter tuning in a predictive nonlinear control method for stable trajectory straight line tracking with a non-holonomic mobile robot. In this method, the focus lies in finding the optimal parameter estimation and to predict the path that the mobile robot will follow for stable trajectory straight line tracking system. The stability control contains three parameters: 1) deflection parameter for the traveling direction of the mobile robot $\left.\left(K_{x}\right) 2\right)$ deflection parameter for the distance across traveling direction of the mobile robot $\left(K_{y}\right)$ and 3) deflection parameter for the steering angle of the mobile robot $\left(K_{\theta}\right)$. Two hundred and seventy three experimental were performed and the results have been analyzed and described herewith. It is found that by using a new optimal parameter tuning in a predictive nonlinear control method derived from the extension of kinematics model, the movement of the mobile robot is stabilized and adhered to the reference posture.
\end{abstract}

Key words: RMSE, Non-holonomic, kinematics model, stable trajectory

\section{INTRODUCTION}

In this study, a new optimal parameter tuning in a predictive nonlinear control method for stable trajectory straight line tracking system with a non-holonomic mobile robot is developed. Stable trajectory straight line tracking control is essential for autonomous mobile robots such as guide robots, security robots and office robots. In the field of mobile robot control, many control schemes for stabilization and trajectory tracking problem have been proposed. Various problems related to the mobile robot's motion control have been studied extensively in recent year. The problems addressed in the literature can be classified into three categories ${ }^{[1]}$ which are posture stabilization, posture tracking and path following.

Posture stabilization addresses stabilizing the mobile robot at a given target point, with desired orientation. This problem lies in controlling three outputs from two inputs. It is noted that the stabilization of wheeled mobile robots with non-holonomic constraints to an equilibrium state is quite difficult as shown by Brockett ${ }^{[2]}$. Nevertheless, several control techniques have been developed such as smooth time-varying control laws ${ }^{[3]}$, discontinuous feedback control law ${ }^{[4]}$ and hybrid feedback control laws ${ }^{[5]}$. No algorithm allows fast, consecutive performance in realistic applications. In realistic implementations, it is difficult to get good performance due to non-holonomic constrain, limitations of motor output, velocity controller performance and system uncertainty.

In posture tracking, a controller is designed to make the mobile robots track the virtual reference mobile robot or a time-parameterized reference. Many authors ${ }^{[6,7]}$ have proposed various control techniques for this problem. However, basic assumption of the posture tracking problem should be noted that the reference posture of mobile robot moves all the time. Hence, stabilization to a fixed posture is not included in the posture tracking problem definition.

Path following control required the mobile robot to converge to and follow a path, without any timing requirements ${ }^{[8,9]}$. It is not well suited for system with strict timing requirement.

The originality of our new optimal parameter tuning in a predictive nonlinear control method for stable trajectory straight line tracking is that, the nonlinear controller is derived from Liapunov stability theory and it is used to control the linear velocity and the angular velocity for locomotion control where the path planner is virtual reference and the problem lies in posture tracking using predictive model to predict the next position of

Corresponding Author: D. Hazry, Artificial Life and Robotics Laboratory, Department of Electrical and Electronic Engineering, Oita University, 700 Oaza Dannoharu, Oita 870-1192, Japan, Tel: +81-97-554-7831, Fax: +81-97-554-7841 
mobile robot in accordance using current velocities of the right wheel and the left wheel. We use the minimal root mean square error (RMSE) in nonlinear controller as measure of control and of quality in determining the optimal parameters to satisfy various design criteria such as stability, performance and robustness.

Kinematics model of mobile robot: The mobile robot has two identical parallel, non-deformable wheels that are controlled by two independent DC motors, thus providing both drive and steering. Additional castors at the front and back of the mobile robot are provided for support. The castors are not considered in obtaining the kinematics and dynamics model. The encoder is used to measure rotation with an accuracy of 80 pulses per revolution. The robot uses $10 \mathrm{~cm}$ diameters wheel. The experiments were carried out on a non-holonomic mobile robot shown in Fig. 1.

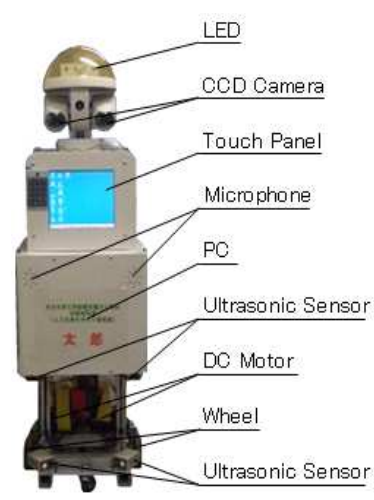

Fig. 1: Non-holonomic mobile robot

A kinematics model is a system of equations that relates the change in kinematics state of a mechanical system to actuator inputs. The system of equations is derived from the geometry of the mechanical system and Newton's laws. It is assumed that the plane of each wheel is perpendicular to the ground and that the contact between the wheels and the ground is pure rolling and non-slipping. The velocity of the center of the mass of the robot is orthogonal to the wheel axis. It is further assumed that the masses and inertias of the wheels are negligible and that the center of mass of the mobile robot is locate in the middle of axis connecting to drive wheels.

The velocity relative to the ground of the right wheel and the left wheel can be expressed as follows:

$$
u_{R}=r \dot{\phi}_{R} \text {, }
$$

$u_{L}=r \dot{\phi}_{L}$,

where $\dot{\phi}_{R}$ and $\dot{\phi}_{L}$ are the angular velocities of the right and the left wheel where "." means the time derivative of function. $r$ is the radius of the wheel. Linear velocity and angular velocity of a mobile robot are related to the wheel velocities, and it can be expressed as Eqs (3) and (4), respectively.
$\mathrm{u}=\left(u_{R}+u_{L}\right) / 2$,

$\omega=\left(u_{R}-u_{L}\right) / 2 d$,

where in Eq. (4) $u_{R}>u_{L}$ and $2 d$ is the width of the mobile robot from the center of the right wheel to the center of the left wheel.

The mobile robot possesses three degree of freedom in its positioning, which can be represented by position and orientation. The vector kinematics state of a mobile robot can be represented by a posture

$p=\left[\begin{array}{l}x \\ y \\ \theta\end{array}\right]$.

By assuming that the wheel do not slide, a non-holonomic constrain on the motion of the mobile robot of the form

$\dot{x} \sin \theta-\dot{y} \cos \theta=0$,

is imposed.

Self positioning presumption-predictive model: Forward kinematics is the technique of predicting a path that differential steering mobile robot will follow. At any instant $x_{c}, y_{c}$ and $\theta_{c}$ posture of the robot's center point are changing based on its speed and orientation. Forward kinematics technique gives the current posture $\left(p_{c}\right)$ of mobile robot with respect to the robot's posture at the beginning of the time period.

$p_{c}=\left[\begin{array}{l}x_{c} \\ y_{c} \\ \theta_{c}\end{array}\right]$,

two wheels velocities, $u_{R}$ and $u_{L}$ where $u_{R}>u_{L}$ taking $\theta$ as a function of time relative distance.

$\theta(t)=\theta_{0}+\frac{\left(u_{R}-u_{L}\right)}{2 d} t$,

where initial orientation of the robot as $\theta(0)=\theta_{0}$. Considering the polar coordinates $(\mathrm{u}(t), \theta(t))$ of the axle center and assuming $x(0)=x_{0}, y(0)=y_{0}$ then

$$
\begin{aligned}
x(t) & =x_{0}+\int_{t-1}^{t} \mathrm{u} \cos \theta d t \\
& =x_{0}+d \frac{\left(u_{R}+u_{L}\right)}{\left(u_{R}-u_{L}\right)}\left[\sin \left(\frac{\left(u_{R}-u_{L}\right)}{2 d} t+\theta_{0}\right)-\sin \left(\theta_{0}\right)\right], \\
y(t) & =y_{0}+\int_{t-1}^{t} \mathrm{u} \sin \theta d t \\
& =y_{0}-d \frac{\left(u_{R}+u_{L}\right)}{\left(u_{R}-u_{L}\right)}\left[\cos \left(\frac{\left(u_{R}-u_{L}\right)}{2 d} t+\theta_{0}\right)-\cos \left(\theta_{0}\right)\right] .
\end{aligned}
$$

Figure 2 shows the predictive model of mobile robot. 


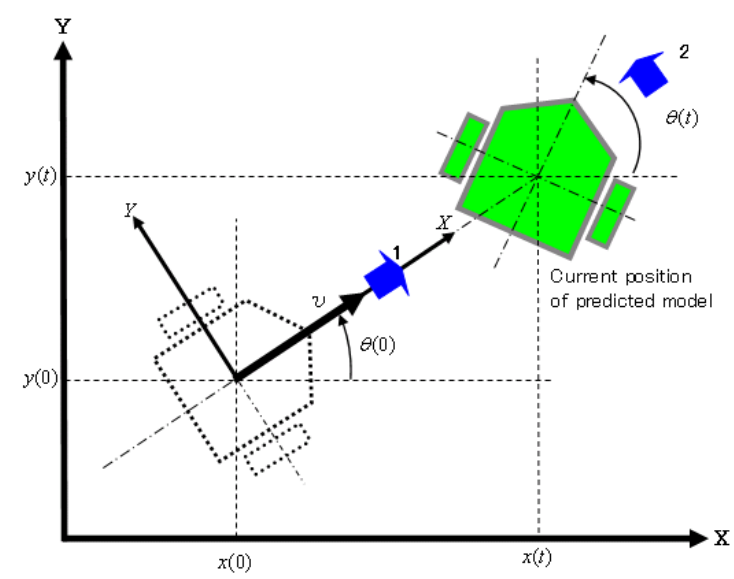

Fig. 2: Predictive model

Mobile robot control system: This task is to construct the wheel velocities input such that the robot tracks the reference posture. In forward kinematics techniques, the unstable running or meandering will result in significant difference between the reference location and the presumption location. Therefore, to meet the above requirement concerning the traveling and direction deflection, it is necessary to decide the optimal constant parameters of the $K_{x}, K_{y}$ and $K_{\theta}$ in Eq. (13). To decide each parameter, the minimal RMSE of deviation in wheel rotations is assumed to be the standard parameter.

In order to simplify the analysis, we consider only situations in which the reference posture is moving on the $X$-axis to the positive direction at a constant velocity. Experiment condition is stated as the mobile robot on the floor with target straight running distance of $500 \mathrm{~cm}$. The reference linear velocity is 80 pulse/sec or equal to $31.415 \mathrm{~cm} / \mathrm{sec}$ at the constant velocity and in order to carry out a wheel rotation as one revolution in one second and the reference angular velocity is $0 \mathrm{rad} / \mathrm{sec}$ in order to ensure the mobile robot running in a straight line. In Eq. (13), $\mathrm{u}_{r}=31.415 \mathrm{~cm} / \mathrm{sec}$ and $\omega_{r}=0 \mathrm{rad} / \mathrm{sec}$. The reference posture $\left(p_{r}\right)$ is defined as follows:

$p_{r}=\left[\begin{array}{c}x_{r} \\ y_{r} \\ \theta_{r}\end{array}\right]$,

where the unit for $x_{r}$ and $y_{r}$ is $\mathrm{cm}$ and the unit for $\theta_{r}$ is rad.

Path error control: Due to nonlinear characteristic and disturbance factors of the mobile robot, there are errors which occurred between the predictive model of current posture and the ideal mobile robot's reference posture. The system is responsible for executing the error posture with minimal deviation. The input to the controller is supplied by the path planner to provide continuous flow of reference posture. The operation of the controller is concerned with knowing the updated current position of predicted model and the mobile robot is tracking the reference postures. The posture control loop tracks the flow of reference postures from the path planner by generating the control variable $u$ and $\omega$ in Eq. (13) for the velocity control loop. The current posture is updated with the forward kinematics technique and the error vector is computed from decomposition of error between the reference posture and the current posture, which is defined as follow:

$p_{e}=\left[\begin{array}{l}x_{e} \\ y_{e} \\ \theta_{e}\end{array}\right]=\left[\begin{array}{ccc}\cos \theta_{c} & \sin \theta_{c} & 0 \\ -\sin \theta_{c} & \cos \theta_{c} & 0 \\ 0 & 0 & 1\end{array}\right]\left[\begin{array}{l}x_{r}-x_{c} \\ y_{r}-y_{c} \\ \theta_{r}-\theta_{c}\end{array}\right]$.

This transformation generates the distance that mobile robot should travel in the forward, lateral and angular directions respectively.

Controller and stability: The following nonlinear feedback control law has been applied

$\left[\begin{array}{c}\mathrm{u} \\ \omega\end{array}\right]=\left[\begin{array}{c}\mathrm{u}_{r} \cos \theta_{e}+K_{x} x_{e} \\ \omega_{r}+\mathrm{u}_{r}\left(K_{y} y_{e}+K_{\theta} \sin \theta_{e}\right)\end{array}\right]$,

it has been proved in $^{[7]}$ that controller from Eq. (12) asymptotically stabilizes Eq. (13) where $K_{x}, K_{y}$ and $K_{\theta}$ are positive constant parameters. It is essential for the system to have the parameter values of $K_{x}, K_{y}$ and $K_{\theta}$ to ensure a functional of the non-oscillatory tracking stabling system without slowing down the response of the mobile robot. Eqs. (14) and (15) describes the relation between $u$ and $\omega$ from Eq. (13) to generate a power wheel steering (PWS) for the right wheel and the left wheel of mobile robot and governing stable movement in trajectory straight line tracking.

$\dot{\phi}_{R}=\frac{\mathrm{u}+d \omega}{r}$,
$\dot{\phi}_{L}=\frac{\mathrm{u}-d \omega}{r}$,

where $d$ is half of distance from the center of the right wheel to the center of the left wheel. In this experiment, the stable trajectory tracking control is defined as a control used to justify robot movement, whereby the robot moves in accordance to the reference posture towards the target within a stated speed and orientation.

Root mean square error: RMSE is an old, proven measure of control and quality ${ }^{[10]}$. RMSE of deviation can be expressed as follows:

$$
\operatorname{RMSE}=\sqrt{\frac{1}{m} \sum_{i=1}^{m}\left(\frac{\sum_{k=1}^{N}\left(e^{(i)}(k)\right)^{2}}{N}\right)},
$$


Am. J. Appl. Sci., 3 (4): 1803-1809, 2006
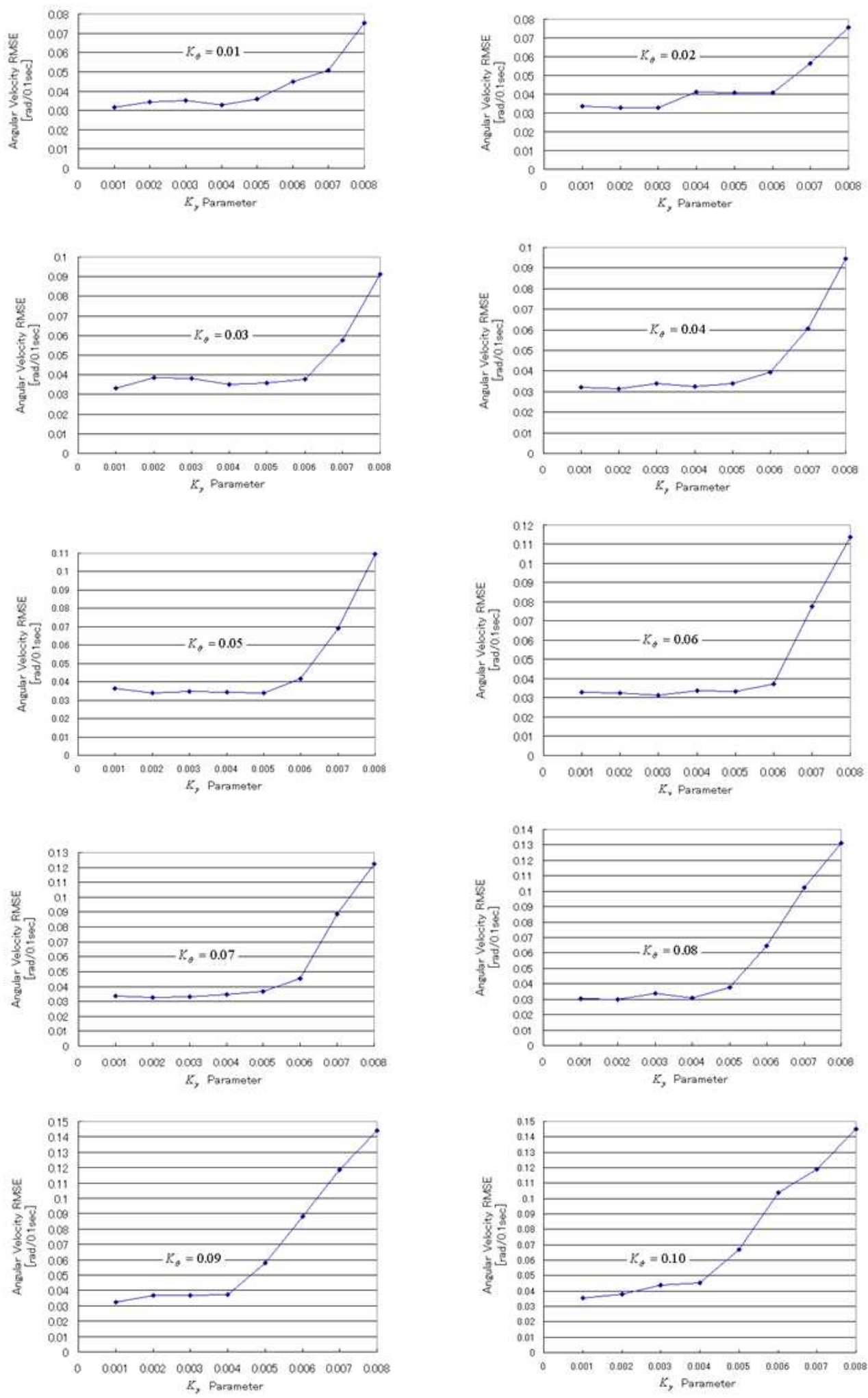

Fig. 3: Angular velocity RMSE of mobile robot with different $K_{\theta}$ and $K_{y}$ parameters 
where $e^{(i)}(k)$ is the linear velocity and the angular velocity error functions of $i$-th experiment define by Eqs. (17) and (18), respectively. $N$ is amount of sample data and $m$ is the numbers of experiments. Angular velocity error function $e_{\omega}$ is used in determining $K_{\theta}$ and $K_{y}$. Linear velocity error function $e_{\mathrm{u}}$ is used in determining $K_{x}$. Angular velocity error function and linear velocity error function can be described as follows:

$e_{\omega}(k)=\omega(k)-\omega_{r}(k)$,

$e_{\mathrm{u}}(k)=\mathrm{u}(k)-\mathrm{u}_{r}(k)$,

where $\omega(k)$ and $u(k)$ are the angular velocity and the linear velocity of the mobile robot in current posture at the $k$-th sampling time defined by Eq. (13). $\omega_{r}(k)$ and $\mathrm{u}_{r}(k)$ are the angular velocity and the linear velocity of the mobile robot in ideal (reference) posture at the $k$-th sampling time which can be describe as follows:

$$
\begin{aligned}
& \theta_{r}=\tan ^{-1} \frac{\dot{x}_{r}}{\dot{y}_{r}}, \\
& \mathrm{u}_{r}=\sqrt{\dot{x}_{r}{ }^{2}+\dot{y}_{r}{ }^{2}}, \\
& \omega_{r}=\dot{\theta}_{r},
\end{aligned}
$$

\section{EXPERIMENTAL RESULT}

Determining $K_{\theta}$ and $K_{y}$ : From Eq. (13), $K_{\theta}$ and $K_{y}$ determines the angular velocity of the mobile robot. Base on preliminary observation if $K_{\theta}$ is more than 0.1 and $K_{y}$ is more than 0.008 the mobile robot will make a start with a high speed movement and it would not be stable. Based on this observation, the experiment is conducted by changing $K_{\theta}$ values from 0.01 until 0.1 and changing $K_{y}$ values from 0.001 until 0.008 . In determining the optimal parameter of $K_{\theta}$ and $K_{y}$ eighty data of the angular velocity RMSE were gathered and analyzed. Each data is an average from three tests. A total of two hundred and forty experiments were conducted.

From the experimental results in Fig. 3, we understand that when $K_{y}$ is more than 0.004 it shows the increasing of the angular velocity RMSE values and as the result the mobile robot do not perform the stable movement in trajectory straight line tracking. Meanwhile when $K_{y}$ is between 0.001 and 0.004 , the mobile robot shows that the angular velocity RMSE are lower and as the result the mobile robot will stably move in trajectory straight line tracking. The lowest value of angular velocity RMSE is the best. We select the lower reading of angular velocity which is $K_{y}$ from 0.01 to 0.04 from Fig. 3 , and plot again the graph as shown in Fig. 4 . The best join selected parameter that perform lower value of the angular velocity

RMSE

are $\left(K_{\theta}=0.08, K_{y}=0.001\right),\left(K_{\theta}=0.08, K_{y}=0.002\right)$, $\left(K_{\theta}=0.06, K_{y}=0.003\right)$ and $\left(K_{\theta}=0.08, K_{y}=0.004\right)$.

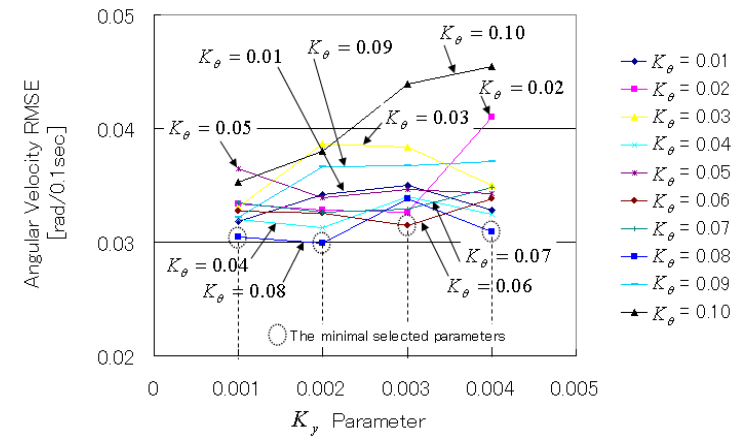

Fig. 4: Minimal angular velocity RMSE of mobile robot with selected parameters $K_{\theta}$ and $K_{y}$

From the best join selected parameters in Fig. 4, the minimal RMSE of deviation in wheel rotations for the right wheel and the left wheel are chosen to be the best positive constant parameter of $K_{\theta}$ and $K_{y}$. The RMSE of deviation in wheel rotations for the right wheel and the left wheel can be expressed as same as Eq. (16) where $e^{(i)}(k)$ is the error function for the right wheel and the left wheel of $i$-th experiment. $e^{(i)}(k)$ for the right wheel and the left wheel are defined as follows:

$$
\begin{aligned}
& e_{R}(k)=\left(\frac{\Delta \phi_{R}}{\Delta t}\right)_{T}-r_{-} o p e(k), \\
& e_{L}(k)=\left(\frac{\Delta \phi_{L}}{\Delta t}\right)_{T}-l_{-} \text {ope }(k),
\end{aligned}
$$

where the numbers of encoder pulses of targets $\left(\frac{\Delta \phi_{R}}{k \Delta}\right)$ and $\left(\frac{\Delta \phi_{L}}{\Delta}\right)$ for the right wheel and the left whdel is equal $\$ 0$ pulses/sec. $r_{-}$ope $(k)$ and $l_{-}$ope $(k)$ are numbers of encoder pulses of the right wheel and the left wheel at the $k$-th sampling time. The experimental result of the RMSE of deviation in wheel rotations for the right wheel and the left wheel are shown in Fig. 5.

From the best join selected parameters in Fig. 5 the lowest RMSE of deviation in wheel rotations for the right wheel and the left wheel is chosen to be the best positive constant parameters of $K_{\theta}$ and $K_{y} . K_{\theta}=0.06$ and $K_{y}=$ 0.003 are decided to be the optimal parameters of $K_{\theta}$ and $K_{\text {. }}$.

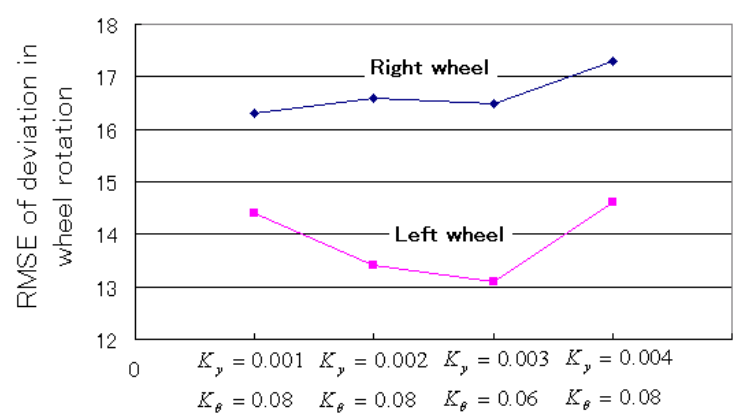

Fig. 5: RMSE of deviation in wheel rotations for $K_{\theta}$ and $K_{y}$ parameters

Determining $K_{x}$ : From Eq. (13), $K_{x}$ determines the linear velocity of the mobile robot. The error margin concerning the traveling distance in $X$ axis direction 
should be as small as possible to make sure the mobile robot asymptotically follow the reference posture in trajectory straight line tracking. From the preliminary observation when $K_{x}$ is more than 0.35 the mobile robot shows high speed of movement. Therefore the experiments are conducted by changing $K_{x}$ parameters values between the ranges of 0.25 to 0.35 . In determining the optimal parameter of $K_{x}$ eleven data of linear velocity RMSE were gathered and analyzed. Each data is an average from three tests. A total of thirty three experiments were conducted. Linear velocity RMSE can be describes by Eqs. (16) and (18). Fig. 6 shows the experimental results.

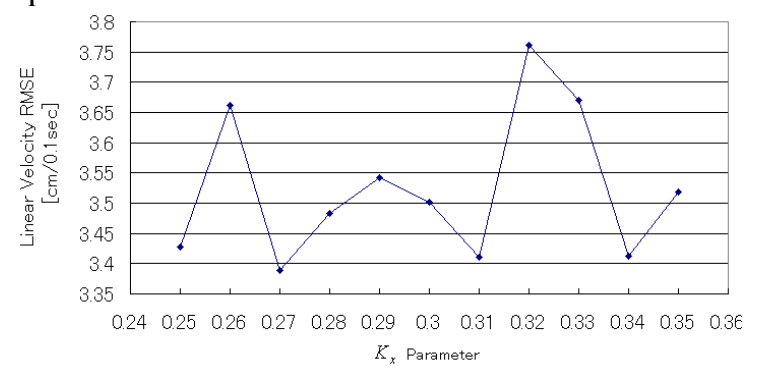

Fig. 6: Linear velocity RMSE of mobile robot with $K_{x}$ parameters value

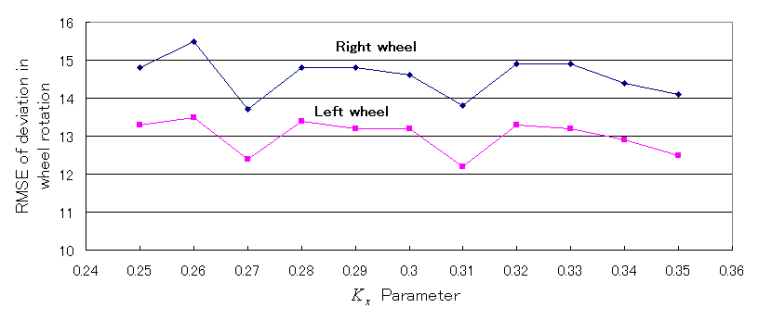

Fig. 7: RMSE of deviation in wheel rotations for $K_{x}$ parameter

The lowest RMSE of deviation in wheel rotations for the right wheel and the left wheel is chosen to be the best positive constant parameters of $K_{x}$. From Fig. 7, there are two minimal similar points. After thorough investigation and experimental observations, $K_{x}=0.27$ is decided to be the optimal parameter of $K_{x}$.

\section{CONCLUSION}

This study has presented experimental results of mobile robot in trajectory straight line tracking with stable control using two velocity controllers accounting for non-holonomic unicycle mobile robot input constraints. Three posture controls for non-holonomic mobile robot are created. Based on the given kinematics design specifications for the desired robot motion tracking, a controller was developed to execute the reference posture. It is shown by Kanayama et al ${ }^{[7]}$ that in Eq. (13) positive constant for the parameters $K_{x}$, $K_{y}$ and $K_{\theta}$ assure that $x_{e}, y_{e}$ and $\theta_{e}$ in Eq. (12) converge to 0 . This involves tuning guideline to obtain the reasonable bounds on the tuning parameters. In order to decide these parameters a series of experiments was setup. The proposed control was demonstrated experimentally at the laboratory environment with target straight running distance is $500 \mathrm{~cm}$. The reference linear velocity is $80 \mathrm{pulse} / \mathrm{sec}$ or equal to $31.415 \mathrm{~cm} / \mathrm{sec}$ at constant velocity in order to carry out a wheel rotation as one revolution in one second. Meanwhile, the reference angular velocity is $0 \mathrm{rad} / \mathrm{sec}$ in order to ensure the mobile robot running in a straight line.

Firstly, we choose to decide $K_{\theta}$ and $K_{y}$ parameters and finally $K_{r}$ parameter by using RMSE explained in section 4.3. From Eq. (13) $K_{\theta}$ and $K_{y}$ determines the angular velocity and $K_{x}$ determines the linear velocity. It was found that by using $K_{\theta}=0.06, K_{y}=0.003$ and $K_{x}=0.27$, the movement of the mobile robot is stabilized, adhere to the reference posture and effective in arriving at the final target in a straight line. It shows that our new method of parameter determination using predictive nonlinear control method for trajectory straight line tracking with three posture control algorithm is effective and feasible. In addition, measuring the position of the mobile robot in the workspace with predictive nonlinear control an indication whether the mobile robot is performing at its best and the confirmation of the parameters will be more accurate.

\section{REFERENCES}

1. Soetanto, D., L. Lapierre and A. Pascoal, 2003. Adaptive, non-singular path-following control of dynamic wheeled robots. Proc. IEEE Conf. on Decision and Control, pp: 1765-1770.

2. Brockett R.W., 1983. Asymptotic stability and feedback stabilization. Differential Geometric Control Theory, Birkhauser, pp: 181-208.

3. Godhavn, J. and O. Egeland, 1997. A Lyapunov approach to exponential stabilization of nonholonomic systems in power form. IEEE Trans. on Automatic Control, 42: 1028-1032.

4. Bloch, A.M., M. Reyhanoglu and N.H. McClamroch, 1992. Control and stabilization of nonholonomic dynamic systems. IEEE Trans. on Automatic Control, 37: 1746-1757.

5. Canudas, C.D.W. and O. Sordalen, 1992. Exponential stabilization of mobile robots with nonholonomic constrain. IEEE Trans. on Automatic Control, 37: 1791-1797.

6. Samson, C. and K.A. Abderrahim, 1991. Feedback control of a nonholonomic wheeled cart in cartesian space. Proc. IEEE, Intl. Conf. on Robotics and Automation, pp: 1136-1141. 
7. Kanayama, Y., Y. Kimura, F. Miyazaki and T. Noguchi, 1991. A stable tracking control method for a non-holonomic mobile robot. Proc. IEEE, Intl Workshop on Intelligent Robots and Systems, pp: 1236-1241.

8. Rio, F.D.D., G. Jimenez, J.L. Sevillano and A.C. Balcells, 2002. A new method for tracking memorized path: Application to unicycle robots. Proceedings of the $10^{\text {th }}$ Mediterranean Conference on Control and Automation.
9. Aguiar, A.P. and J.P. Hespenha, 2004. Logic-based switching control for trajectory and path-following of underactuated autonomous vehicles with parametric modeling uncertainty. Proc. Am. Control Conf., 4: 3004-3010.

10. Battaglia, G.J., 1996. Mean square error. AMP J. Technol., 5: 31-36. 